\title{
A useful exercise test for detecting leg malperfusion due to aortic dissection
}

\author{
Baku Takahashi $^{1}$ and Keiji Kamohara ${ }^{1}$ \\ ${ }^{1}$ Saga University Hospital
}

March 13, 2021

\begin{abstract}
We herein report a case of leg malperfusion caused by dynamic obstruction after aortic dissection diagnosed by the exercise ankle brachial pressure index test that could not be diagnosed solely by examining the symptoms and investigations at rest. This case suggests that exercise can be a key factor in the diagnosis of this complication. Furthermore, blood pressure elevation can be an exacerbating factor in dynamic obstruction. We recommend conducting an aggressive evaluation of the symptoms and medical examinations not only at rest but also under stress.
\end{abstract}

\section{INTRODUCTION}

The diagnosis of leg malperfusion caused by dynamic obstruction after aortic dissection is difficult because the dissection flap remains unstable and often causes changes in symptoms. We herein report a case of leg malperfusion caused by dynamic obstruction after aortic dissection diagnosed by the exercise ankle brachial pressure index (Ex-ABPI) test. We believe that this test is more objective and useful than the methods used for making a diagnosis in previous reports. ${ }^{1,2}$ Furthermore, the present case demonstrated a unique feature of the relationship between leg malperfusion and blood pressure (BP).

\section{CASE PRESENTATION}

A 59-year-old man underwent arch replacement using the frozen elephant trunk technique for acute type A aortic dissection. The postoperative course was uneventful. However, two weeks later, he complained of numbness and pain in his right leg during movement. The symptoms were nonspecific, and the resting ABPI was over 1.00 in both legs. Contrast-enhanced computed tomography (CT) revealed that the false lumen was patent with an entry at the Th8 level (Fig. 1a), and an intimal flap extended to the left common iliac artery, although the true lumen was maintained and not severely compressed at the periphery.

The Ex-ABPI test (tilt angle $12 \%$, treadmill set at $2.4 \mathrm{~km} / \mathrm{h}$ ) was performed. Five minutes later, the test was stopped due to limb pain. Although the right ABPI at rest was 1.00 before exercise (at left brachial BP $127 / 69 \mathrm{mmHg}$ ), it hit the lowest point of 0.23 at 1 minute after exercise (at left brachial BP 146/78 $\mathrm{mmHg}$ ) and recovered to 0.84 at 5 minutes later (at left brachial BP 126/64 mmHg) (Fig. 2a). The left ABPI and his heart rate remained unchanged during the examination. Thoracic endovascular aortic repair (TEVAR) was performed to occlude the proximal entry site (Fig. 1b). Postoperatively, the symptoms of lower limb pain disappeared completely, and Ex-ABPI tests showed improvement under the same condition at which he had experienced pain earlier (Fig. 2b).

\section{Comment}

Leg malperfusion after aortic dissection occurs in $10.3 \%$ of cases with aortic dissection. ${ }^{3}$ There are two pathophysiological mechanisms of malperfusion: static obstruction and dynamic obstruction. ${ }^{4}$ Static obstruction 
can cause malperfusion because the branch vessels dissociate, and stenosis or occlusion occurs due to thrombus. This condition can be easily diagnosed by CT. In contrast, dynamic obstruction causes malperfusion because the false lumen expands, and the dissection flap covers the vessel origin. The diagnosis of this complication is difficult because the dissection flap usually remains unstable and moves easily. Previous reports have found that postural change ${ }^{1}$ and exercise echography ${ }^{2}$ are helpful for diagnosing leg malperfusion due to dynamic obstruction. In our case, since postural change did not cause any symptoms, and given that echography requires a high level of skill, the Ex-ABPI test was performed. This examination demonstrated that the patient's right ABPI fell significantly after exercise, which was helpful for detecting this complication. To our knowledge, this is the first report of leg malperfusion caused by dynamic obstruction after aortic dissection being diagnosed by the Ex-ABPI test.

Of note, the Ex-ABPI test suggested an interesting finding of a negative correlation between leg malperfusion and BP. In experimental models, the entry size and physiological factors have a complex influence true lumen collapse. ${ }^{5}$ However, what aggravates dynamic obstruction in vivo remains unclear. In the present case, BP elevation may have been a factor that exacerbated the dynamic obstruction. From that perspective, leg malperfusion was also exacerbated at BP elevation and restored at BP reduction in a previous report. ${ }^{2}$ It is difficult to explain the exact mechanism underlying this unique feature, but it may involve an increase in the cardiac output because of exercise resulting in an increased inflow into the false lumen, with the false lumen consequently expanding and compressing the true lumen further; alternatively, the stenosis of the true lumen may have been exacerbated by the contraction of the aorta itself by exercise, which diminished the blood flow to his right leg and exacerbated peripheral malperfusion. Even after TEVAR, the slight decrease in the right ABPI by BP elevation after exercise can be attributed to residual re-entry at the peripheral side.

An important finding from this case is that dynamic obstruction should not be ruled out, even if the symptoms and examinations are not marked at rest. Stress can be a key factor in the diagnosis of this complication. However, the degree of stress in such cases remains unclear, and symptoms can be caused either by postural change alone, ${ }^{1}$ or by some degree of stress as observed in both the previous case ${ }^{2}$ and ours. Exercise echography may also be useful, but the Ex-ABPI test has more advantages, such as a low cost, low invasiveness, high reproducibility, ease of testing, and ease of interpreting test results. Lower limb ischemia caused by aortic dissection is a predictive factor for death and visceral ischemia. ${ }^{6}$ Therefore, we recommend an aggressive evaluation of the symptoms and the performance of medical examinations not only at rest but also under stress, with an increase in the BP required to make an accurate diagnosis of leg malperfusion following aortic dissection.

\section{ACKNOWLEDGEMENTS}

We would like to thank Editage (www.editage.com) for the English language editing.

\section{CONFLICT OF INTERESTS}

The authors declare that there are no conflict of interests.

\section{INSTITUTIONAL REVIEW BOARD APPROVAL OF WAIVER}

No need for IRB approval for this study.

\section{PATIENT CONSENT STATEMENT}

Consent was not obtained and waived.

\section{DATA AVAILABILITY STATEMENT}

Data available on request.

\section{References}

1. Nakahira A, Ogino H, Matsuda H, et al. Postural change causing leg malperfusion resulting from expansion of a patent false lumen in type B aortic dissection. J Thorac Cardiovasc Surg 2007;134:1046-1047. 
2. Yoshimuta T, Tsuneto A, Okajima T, et al. Doppler ultrasound diagnosis of transient leg malperfusion caused by dynamic obstruction in a patient with chronic aortic dissection. Echocardiography 2019;36:189191.

3. Charlton-Ouw KM, Sandhu HK, Leake SS, et al. Need for Limb Revascularization in Patients with Acute Aortic Dissection is Associated with Mesenteric Ischemia. Ann Vasc Surg 2016;36:112-120.

4. Williams DM, Lee DY, Hamilton BH, et al. The dissected aorta: part III. Anatomy and radiologic diagnosis of branch-vessel compromise. Radiology 1997;203:37-44.

5. Chung JW, Elkins C, Sakai T, et al. True-lumen collapse in aortic dissection: part I. Evaluation of causative factors in phantoms with pulsatile flow. Radiology 2000;214:87-98.

6. Henke PK, Williams DM, Upchurch GR Jr, et al. Acute limb ischemia associated with type B aortic dissection: clinical relevance and therapy. Surgery 2006;140:532-539.

\section{Figure legends}

Figure 1. CT findings: a, Before thoracic endovascular aortic repair (TEVAR), the false lumen was patent, and the arrow indicates the entry at the Th8 level. b, After TEVAR, the entry was closed completely. The false lumen was thrombosed around the entry but patent due to retrograde flow from re-entry.

Figure 2. The relationship between the ABPI and the physiological factors before and after exercise. a, Before TEVAR, the right ABPI was 1.00 at rest at a left brachial BP $127 / 69 \mathrm{mmHg}$, but it dropped significantly to 0.23 at 1 minute after exercise at a left brachial $\mathrm{BP} 146 / 78 \mathrm{mmHg}$ and recovered to 0.84 at 5 minutes after exercise at a left brachial BP 126/64 mmHg. The left ABPI and HR remained stable, regardless of exercise. b, After TEVAR, the right ABPI significantly improved throughout the test. (TEVAR, thoracic endovascular aortic repair; ABPI, ankle brachial pressure index; Ex, exercise; min, minute; sBP, systolic blood pressure; dBP, diastolic blood pressure; HR, heart rate; Rt, right; Lt, left.)

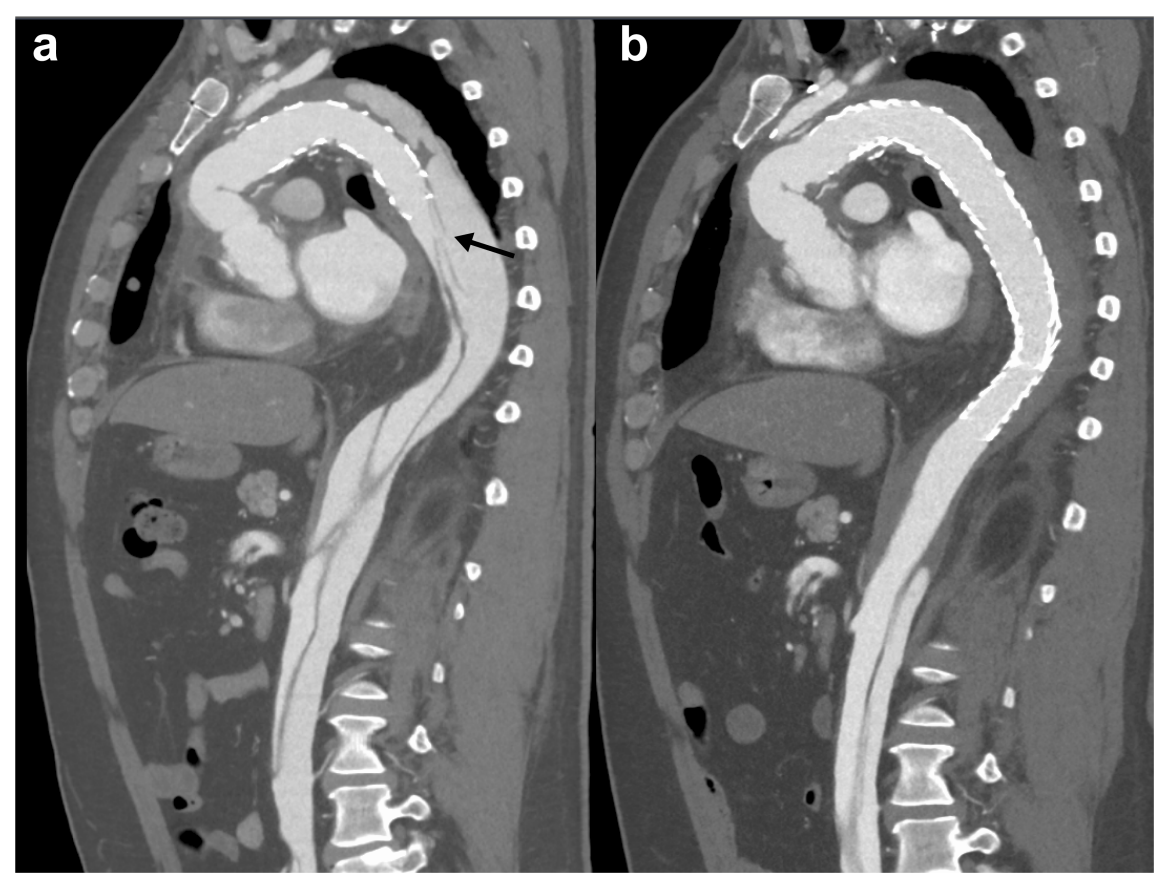


a Before TEVAR

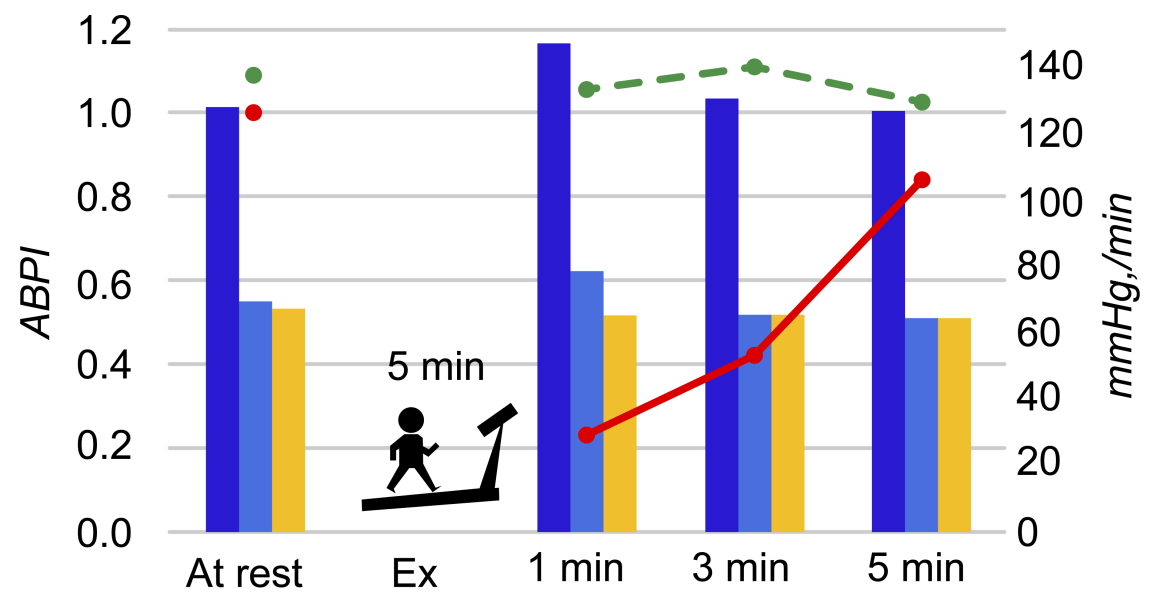

$\square$ Brachial sBP Brachial dBP $\square$ HR $\rightarrow$ Rt. $\bullet-$ Lt.

b

After TEVAR

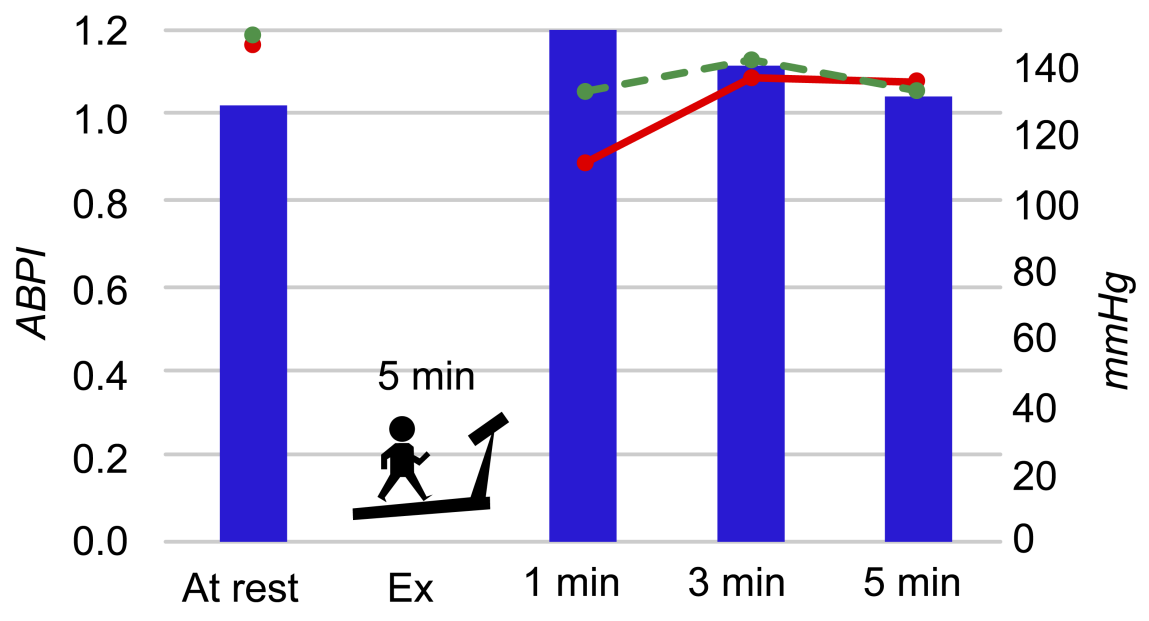

Brachial sBP $\rightarrow$ Rt. $\cdots-$ Lt. 